\title{
A cautionary tale of cross-contamination among plasmids from commercial suppliers
}

Jinli Sun ${ }^{1,2}$, Yaping Tian ${ }^{3}$, Yingying Du², Zhenzhen Wang ${ }^{4}$, Guodong Zhao*,2, Yong Ma*,2 \& Minxue Zheng*,2

\section{ABSTRACT}

Many researchers have switched to purchasing their desired plasmids from commercial suppliers to save time and resources, as we did for 17 high-risk human papillomavirus plasmids. To our surprise, they were shown to be cross-contaminated with one another. Comparison between the production schedule and the pattern of contaminations proved that this contamination occurred during the production process, which was also shown for another two sets of commercial plasmids. Our experience indicates that the absolute purity of plasmids obtained from external sources cannot be guaranteed. Extreme caution should be exercised, especially when such plasmids are used for human gene therapies and DNA vaccines, where even a minute amount of contamination may pose significant risks to patients.

\section{METHOD SUMMARY}

Cross-contamination among commercial plasmids was discovered by performing real-time quantitative PCR reactions specific to individual plasmids on all plasmids, and the identities of the suspected contamination determined by Sanger sequencing of the PCR products and subsequent BLAST searches with the sequencing results as query sequences. The contaminated plasmids were transformed into Eschericia coli competent cells and single transformants were amplified by overnight culture for plasmid repurification. To minimize the possibility of cross-contamination, all equipment used for plasmid purification was thoroughly cleaned both before and after purification, and only one plasmid was repurified each day.

\section{KEYWORDS}

biotechnology $\cdot$ cross-contamination $\cdot$ plasmid - purity • quantitative PCR

'School of Life Sciences, University of Science \& Technology of China, Hefei, Anhui 230027, China; ${ }^{2}$ Suzhou Institute of Biomedical Engineering \& Technology, Chinese Academy of Sciences, Suzhou, Jiangsu 215163, China; ${ }^{3}$ The First Hospital of Jilin University, Changchun, Jilin 130021, China; ${ }^{4}$ School of Medical Technology, Xuzhou Medical University, Xuzhou, Jiangsu 221004, China; *Authors for correspondence: gddn89429@hotmail.com, mayong@sibet.ac.cn, minxue. zheng@sibet.ac.cn

BioTechniques 68: 14-21 (January 2020) 10.2144/btn-20190018
Plasmids are a class of transmissible, extrachromosomal genetic elements that were first identified in bacteria [1]. Naturally occurring plasmids mediate a variety of biological functions including bacterial conjugation, transfer of disease-causing virulence genes and transfer of resistance genes to various detrimental environmental factors, such as antibiotics, radiation and heavy metals. With the discovery of restriction enzymes [2,3] and subsequent development of recombinant DNA technology [4], plasmids have become versatile tools in basic biological research and biotechnology development for biomedical, agricultural and industrial applications [5-11]. With the advent of de novo DNA synthesis [12] and the decreasing cost and rising efficiency of relevant technologies, more and more researchers have switched to de novo synthesis of their desired plasmids to save resources and time required for traditional cloning methods $[13,14]$. In addition, nearly every supplier of biological research reagents worldwide is now providing this service to the scientific community.

Like every other reagent, absolute purity is desired for plasmids from commercial sources as well as other research organizations. Even though, depending on the goals, some research projects may tolerate reagents with very low levels of contamination by unintended components such as buffer solutions, protein preparations and even plasmids, absolute purity is necessary for other applications. The areas where nucleic acid contamination may cause serious consequences include molecular diagnosis, gene therapy and plasmid vaccines developed to detect, treat and/or prevent human diseases $[15,16]$. PCR technology has been widely used for the molecular diagnosis of various diseases, where its high sensitivity is desirable [17]. However, high sensitivity of PCR could also lead to false-positive diagnostic results as a minute amount of nucleic acid contamination may result in misdiagnosis $[18,19]$. On the other hand, even extremely low levels of contamination in gene therapy vectors or plasmid vaccines could pose significant risks to the health and safety of patients $[15,16]$. It has been shown that, despite the extremely low likelihood, rare events have occurred in clinics [20]. Therefore, the approaches to detect and minimize nucleic acid contamination for these applications have been extensively discussed in the literature [21-23]. However, except for double-transformation artifacts [24], to our knowledge no published studies have touched on the possibility that plasmids used for these applications may be contaminated by other unwanted plasmids.

In this study, we reported multiple incidences of proven cross-contamination among the same batch of plasmids purchased from commercial suppliers, which calls for more attention to be paid to plasmid purity for applications where absolute purity is paramount.

\section{MATERIALS \& METHODS CervilHPV test \& HR-HPV Genotyping Assay}

The CerviHPV test (GWP Biotechnology Co. Ltd., Suzhou, China) is a TaqMan-based multiplex quantitative PCR (qPCR)-based high-risk human papillomavirus (HR-HPV) detection assay [25]. It simultaneously detects 
Table 1. Standard curve experiment results for HPV18 reference plasmid by CerviHPV qPCR assay.

\begin{tabular}{|c|c|c|c|}
\hline \multirow{2}{*}{$\begin{array}{l}\text { Plasmid concentration } \\
\text { (copies/reaction) }\end{array}$} & \multirow{2}{*}{ PCR replicates (n) } & \multicolumn{2}{|c|}{ Mean Ct \pm SD } \\
\hline & & HPV18 & $12 \mathrm{HPV} \ddagger$ \\
\hline $1.0 \times 10^{8}$ & 3 & $14.3 \pm 0.2$ & $34.6 \pm 0.4$ \\
\hline $1.0 \times 10^{7}$ & 3 & $18.1 \pm 0.3$ & $38.1 \pm 0.4$ \\
\hline $1.0 \times 10^{6}$ & 3 & $21.7 \pm 0.3$ & $41.8 \pm 0.8$ \\
\hline $1.0 \times 10^{5}$ & 3 & $25.3 \pm 0.2$ & $45.8 \pm 4.2$ \\
\hline $1.0 \times 10^{4}$ & 3 & $28.8 \pm 0.4$ & I \\
\hline $1.0 \times 10^{3}$ & 3 & $32.6 \pm 0.5$ & I \\
\hline $1.0 \times 10^{2}$ & 6 & $36.7 \pm 0.6$ & I \\
\hline $1.0 \times 10^{1}$ & 12 & $39.4 \pm 0.9$ & I \\
\hline $1.0 \times 10^{0}$ & 12 & $38.7^{+}$ & I \\
\hline
\end{tabular}

14 HR-HPV types - HPV16, 18, 31, 33, 35, $39,45,51,52,56,58,59,66$ and $68-$ in a single reaction tube. HPV16 and 18 are each detected by an individual fluorescent channel, whereas the third channel reports the presence of any one or combination of the remaining $12 \mathrm{HR}$-HPV types (abbreviated as 12 HPV types). The fourth channel detects a human gene as the cellular control.
HR-HPV Genotyping Assay (GWP Biotechnology Co. Ltd.) is a TaqManbased APCR assay that genotypes 12HPV types [25]. It employs three five-channel multiplex QPCR reactions, each of which can individually identify four 12HPV types. In each of these reactions, each HR-HPV type is recognized by one fluorescent channel, whereas the fifth channel detects the cellular control.

\section{Other $\mathrm{qPCR}$ reactions}

TaqMan-based qPCR reactions for 13 bacterial diarrhea-causing virulence genes will be described elsewhere [submitted manuscript]. For another ongoing project, we have developed TaqMan-based GPCR reactions to detect human $\angle L 7 B$ and $C X C L 8$ cDNA. All qPCR assays used in this study were performed on $\mathrm{ABI} 7500$ Fast instrument (Thermo Fisher Scientific, MA,

\section{Table 2. Genotyping qPCR experiment results with HPV18 plasmid as PCR template at a concentration of $1.0 \times 10^{8}$ copies/reaction.}

\section{HR-HPV channel}

\section{Mean Ct \pm SD}

\begin{tabular}{|l|l|l|}
\hline 31 & $35.8 \pm 0.0$ & HPV31 \\
\hline 33 & $/$ & NA \\
\hline 35 & $37.2 \pm 1.2$ & OP \\
\hline 39 & $38.5 \pm 0.4$ & OP \\
\hline 45 & $/$ & NA \\
\hline 51 & $32.2 \pm 0.3$ & HPV51-1 \\
\hline 52 & $35.6 \pm 0.1$ & HPV52-1 \\
\hline 56 & $38.7 \pm 0.1$ & HPV18 \\
\hline 58 & $39.8 \pm 0.4$ & SC \\
\hline 59 & $38.3 \pm 0.4$ & HPV59 \\
\hline 66 & $37.2 \pm 0.8$ & HPV66 \\
\hline 68 & $/$ & NA \\
\hline $\begin{array}{l}\text { TSequencing result: NA: Not applicable; OP: Overlapping peaks; SC: Scrambled chromatogram. } \\
\text { HR-HPV: High-risk human papillomavirus. }\end{array}$ & \\
\hline
\end{tabular}


USA) according to the manufacturer's instructions.

\section{Standard curve experiment}

All HR-HPV reference plasmids were first diluted to a concentration of $1.0 \times 10^{8}$ copies/reaction. The resulting plasmids then underwent eight tenfold serial dilutions to generate a concentration gradient of $10^{7}$ to 1 copies/reaction. The CerviHPV test was performed with the concentration gradient solutions to generate standard curves.

\section{Sanger sequencing}

The qPCR products of reactions with unexpected amplification signals were first purified by the PCR Product Purification Kit (Sangon Biotech Co. Ltd., Shanghai, China) and the purified products were subsequently sequenced by Genewiz Inc. (Suzhou, China), with specific primers for the contaminating templates [25]. The resulting DNA sequences were queried on the BLAST website [26] to determine their identities.

\section{Repurification of contaminated}

\section{plasmids}

To generate pure plasmid preparations, the contaminated plasmids underwent a repurification process one by one to minimize the possibility of cross-contamination between different plasmids. Escherichia coli competent cells (Tiangen Biotech Co. Ltd., Beijing, China) were transformed with one contaminated plasmid and spread onto a Luria-Bertani broth agar plate at such a density that less than 100 colonies formed on the plate after incubation at $37^{\circ} \mathrm{C}$ overnight. On the second day, $4 \mathrm{ml}$ liquid LB medium was inoculated with a single colony and cultured at $37^{\circ} \mathrm{C}$ with shaking overnight. On the third day, all equipment to be used for plasmid purification, including pipettes, centrifuges, mixers, benchtops and tube racks, were thoroughly disinfected by bleach and cleaned with $75 \%$ ethanol before plasmid purification. Plasmid was subsequently purified from the overnight culture with the AxgPrep Plasmid DNA Extraction Kit (Corning Inc., NY, USA) according to the manufacturer's instructions. Afterwards, all the equipment used in the purification process was again thoroughly disinfected with bleach and cleaned with $75 \%$ ethanol. To further minimize cross-contamination, only one plasmid was repurified each day,

\begin{tabular}{|c|c|c|c|c|c|c|c|c|c|c|c|c|c|c|c|c|c|c|c|}
\hline$\frac{\infty}{\stackrel{\infty}{\circ}}$ & & 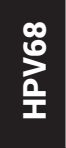 & - & - & - & 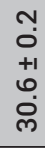 & - & - & - & - & - & - & - & - & - & $\begin{array}{l}N \\
0 \\
+1 \\
o \\
\text { mे } \\
m\end{array}$ & - & $\begin{array}{l}0 \\
0 \\
+1 \\
10 \\
\dot{m} \\
\dot{n}\end{array}$ & \\
\hline $\begin{array}{l}0 \\
\text { ? } \\
4 \\
0 \\
0 \\
0\end{array}$ & & 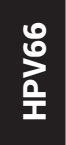 & - & 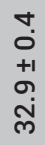 & - & $\begin{array}{l}m \\
0 \\
+1 \\
\stackrel{+}{n} \\
\stackrel{m}{m}\end{array}$ & $\begin{array}{l}0 \\
0 \\
+1 \\
m \\
\tilde{N} \\
\tilde{m}\end{array}$ & - & - & 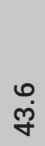 & - & 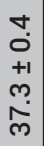 & - & $\begin{array}{l}\hat{0} \\
0 \\
+1 \\
\text { L } \\
\text { مें }\end{array}$ & - & $\begin{array}{l}0 \\
\stackrel{-}{-} \\
+1 \\
\sim \\
\infty \\
\infty \\
m\end{array}$ & 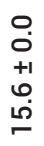 & $\begin{array}{l}10 \\
0 \\
+1 \\
\hat{N} \\
\hat{m}\end{array}$ & \\
\hline 을 & & $\begin{array}{l}\text { 용 } \\
\text { 올 }\end{array}$ & - & - & - & $\begin{array}{l}\infty \\
0 \\
+1 \\
+1 \\
\infty \\
\dot{m}\end{array}$ & $\begin{array}{l}+ \\
0 \\
+1 \\
\infty \\
\infty \\
\infty\end{array}$ & - & - & - & - & $\begin{array}{l}\hat{0} \\
0 \\
+1 \\
\infty \\
\infty \\
\dot{m}\end{array}$ & - & 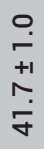 & - & $\begin{array}{l}\check{0} \\
+1 \\
\dot{+} \\
\dot{m}\end{array}$ & $\begin{array}{l}+ \\
0 \\
+1 \\
0 \\
0 \\
\end{array}$ & $\begin{array}{l}0 \\
0 \\
+1 \\
+1 \\
\infty \\
\infty \\
\infty\end{array}$ & \\
\hline 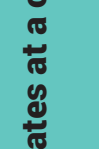 & & 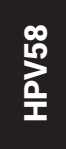 & - & $\begin{array}{l}\infty \\
0 \\
+1 \\
+1 \\
0 \\
\infty \\
\infty \\
\infty\end{array}$ & - & $\begin{array}{l}\dot{+} \\
\dot{0} \\
+1 \\
\dot{0} \\
\dot{j}\end{array}$ & $\begin{array}{l}+ \\
0 \\
+1 \\
\square \\
\square\end{array}$ & - & - & $\begin{array}{l}\bar{m} \\
+1 \\
10 \\
\stackrel{+}{+}\end{array}$ & - & $\begin{array}{l}\overline{0} \\
+1 \\
+ \\
\bar{\nabla}\end{array}$ & - & - & $\begin{array}{l}0 \\
0 \\
+1 \\
0 \\
\dot{+} \\
\dot{I}\end{array}$ & $\begin{array}{l}\infty \\
0 \\
+1 \\
+1 \\
+\infty \\
\infty\end{array}$ & $\begin{array}{l}10 \\
0 \\
+1 \\
0 \\
0 \\
\dot{v}\end{array}$ & $\begin{array}{c}N \\
0 \\
+1 \\
N \\
\text { ஸे } \\
\text { ले }\end{array}$ & \\
\hline 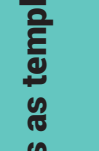 & (ब) & 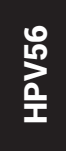 & - & - & - & 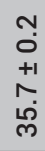 & 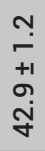 & - & - & - & - & $\begin{array}{l}N \\
0 \\
+1 \\
\infty \\
\infty \\
\stackrel{m}{m}\end{array}$ & - & $\begin{array}{l}\overrightarrow{0} \\
+1 \\
\stackrel{+}{n} \\
\dot{m}\end{array}$ & - & $\begin{array}{l}\sigma \\
\stackrel{+}{+} \\
+1 \\
\\
\dot{m}\end{array}$ & $\begin{array}{l}+ \\
0 \\
+1 \\
+ \\
\dot{m} \\
\dot{m}\end{array}$ & $\begin{array}{l}0 \\
0 \\
+1 \\
+0 \\
0 \\
0 \\
\text { ले }\end{array}$ & \\
\hline 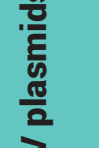 & 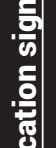 & §̊ & - & $\stackrel{\circ}{\dot{f}}$ & - & $\begin{array}{l}\tilde{N} \\
0 \\
+1 \\
m \\
m \\
m\end{array}$ & - & - & $\begin{array}{l}\stackrel{9}{0} \\
0 \\
+1 \\
\text { N } \\
\text { மn }\end{array}$ & ্ָণ & - & $\begin{array}{l}0 \\
0 \\
+1 \\
\circ \\
\dot{m} \\
\dot{r}\end{array}$ & $\begin{array}{c}\check{\sigma} \\
+1 \\
+1 \\
\sigma \\
\end{array}$ & $\begin{array}{l}\hat{\sim} \\
+1 \\
+1 \\
\stackrel{+}{+}\end{array}$ & 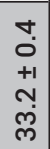 & $\begin{array}{l}\sigma \\
0 \\
+1 \\
\infty \\
0 \\
\dot{m}\end{array}$ & - & $\begin{array}{l}\overline{0} \\
+1 \\
m \\
m \\
\text { Lे }\end{array}$ & \\
\hline 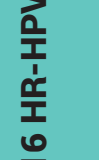 & 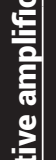 & $\frac{p}{p}$ & - & 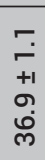 & - & $\begin{array}{l}\tilde{0} \\
+1 \\
\text { oे } \\
\text { N }\end{array}$ & $\begin{array}{l}N \\
0 \\
+1 \\
+\infty \\
+ \\
+\end{array}$ & - & - & $\begin{array}{l}\tilde{O} \\
+1 \\
\stackrel{+}{N} \\
\stackrel{N}{ }\end{array}$ & $\begin{array}{l}0 \\
0 \\
+1 \\
10 \\
\stackrel{1}{1} \\
\end{array}$ & 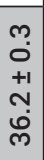 & - & $\begin{array}{l}\infty \\
\Gamma \\
+1 \\
0 \\
\sigma \\
\sigma\end{array}$ & - & $\begin{array}{l}\text { L } \\
0 \\
+1 \\
+1 \\
\text { L } \\
\text { Ln }\end{array}$ & 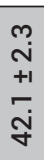 & 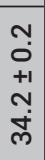 & \\
\hline 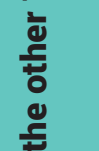 & 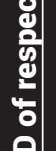 & 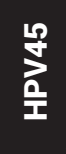 & - & - & $\begin{array}{l}0 \\
0 \\
+1 \\
0 \\
0 \\
m\end{array}$ & - & - & $\begin{array}{l}\widetilde{0} \\
+1 \\
\stackrel{+}{N} \\
\stackrel{N}{-}\end{array}$ & $\begin{array}{l}0 \\
0 \\
+1 \\
+ \\
\check{C} \\
\mp\end{array}$ & - & - & - & $\begin{array}{l}+ \\
0 \\
+1 \\
\infty \\
\text { సे }\end{array}$ & - & - & - & - & - & \\
\hline $\begin{array}{l}\frac{c}{3} \\
\frac{1}{3} \\
\frac{0}{3}\end{array}$ & 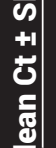 & ฉి & - & $\begin{array}{l}\stackrel{+}{0} \\
+1 \\
\stackrel{+}{N} \\
\ddot{n}\end{array}$ & - & $\begin{array}{l}m \\
0 \\
+1 \\
+ \\
\stackrel{m}{\rho}\end{array}$ & $\begin{array}{l}0 \\
0 \\
+1 \\
0 \\
\dot{m} \\
\ddot{n}\end{array}$ & - & - & 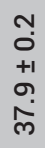 & - & $\begin{array}{l}\overline{0} \\
+1 \\
\overline{+1} \\
\text { ம் }\end{array}$ & - & $\begin{array}{l}\text { م) } \\
0 \\
+1 \\
\text { N̦ } \\
\hat{m}\end{array}$ & - & $\begin{array}{l}\text { - } \\
+1 \\
+ \\
\text { ம் } \\
\text { மे }\end{array}$ & $\begin{array}{l}m \\
0 \\
+1 \\
0 \\
0 \\
\bar{m}\end{array}$ & $\begin{array}{l}\stackrel{N}{0} \\
0 \\
+1 \\
\text { ñ. } \\
\text { }\end{array}$ & \\
\hline 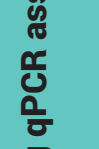 & & 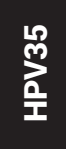 & - & - & - & $\begin{array}{l}\tilde{\sigma} \\
+1 \\
o \\
\stackrel{+}{N}\end{array}$ & 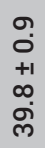 & - & - & - & - & 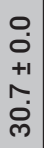 & - & 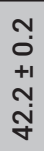 & - & $\begin{array}{l}+ \\
0 \\
+1 \\
+ \\
\dot{m}\end{array}$ & - & 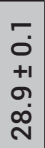 & \\
\hline ํㅡㄹ & & $\frac{m}{\gtrless}$ & - & - & $\begin{array}{l}0 \\
0 \\
+1 \\
+ \\
m \\
m \\
\end{array}$ & - & - & $\begin{array}{l}\check{0} \\
+1 \\
\hat{N} \\
\dot{m}\end{array}$ & - & - & - & - & - & - & - & - & - & - & 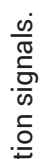 \\
\hline 을 & & $\frac{n}{2}$ & - & $\begin{array}{l}\sigma \\
0 \\
+1 \\
0 \\
\end{array}$ & - & $\begin{array}{l}m \\
0 \\
+1 \\
0 \\
\text { ம. } \\
\text { ले }\end{array}$ & $\begin{array}{l}m \\
\sim \\
+1 \\
i \\
\dot{m} \\
\dot{m}\end{array}$ & - & - & - & - & $\begin{array}{l}0 \\
0 \\
+1 \\
0 \\
\dot{j} \\
\text { ले }\end{array}$ & - & 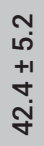 & - & - & $\begin{array}{l}m \\
0 \\
+1 \\
\text { N } \\
\text { N่}\end{array}$ & 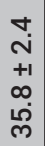 & 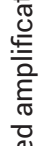 \\
\hline 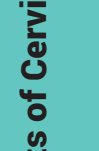 & & $\stackrel{\infty}{\gtrless}$ & - & - & - & $\begin{array}{l}- \\
0 \\
+1 \\
0 \\
\dot{m} \\
m\end{array}$ & - & - & - & $\begin{array}{l}9 \\
0 \\
+1 \\
\sigma \\
0 \\
\dot{+}\end{array}$ & - & $\begin{array}{l}m \\
0 \\
+1 \\
+1 \\
\stackrel{m}{m}\end{array}$ & - & - & - & $\begin{array}{l}\stackrel{+}{-} \\
+1 \\
\\
\hat{m}\end{array}$ & - & $\begin{array}{l}\sim \\
0 \\
+1 \\
i \\
\text { Ln } \\
\text { ले }\end{array}$ & 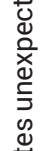 \\
\hline 홍 & & $\sum_{e}^{\bullet}$ & $\begin{array}{l}\text { Na } \\
+1 \\
+1 \\
\dot{+} \\
\ddot{r}\end{array}$ & - & - & $\begin{array}{l}\stackrel{L}{\infty} \\
\stackrel{\infty}{+}\end{array}$ & $\begin{array}{l}0 \\
0 \\
+1 \\
0 \\
0 \\
0\end{array}$ & - & - & 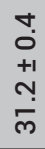 & - & 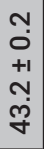 & - & - & - & \begin{tabular}{l}
\multirow{0}{0}{} \\
+1 \\
N \\
$\dot{m}$
\end{tabular} & $\begin{array}{l}m \\
0 \\
+1 \\
m \\
\infty \\
\infty \\
m\end{array}$ & - & 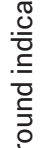 \\
\hline 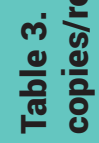 & 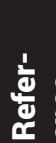 & 送 & $\begin{array}{l}\stackrel{0}{2} \\
\text { 몯 }\end{array}$ & 辛 & 总 & 总 & 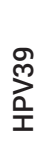 & $\begin{array}{l}\text { i⿱ } \\
\text { \& } \\
\text { 모 }\end{array}$ & \begin{tabular}{l} 
N \\
L⿱ \\
\multirow{2}{Z}{} \\
모
\end{tabular} & 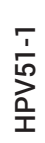 & $\begin{array}{l}\frac{N}{1} \\
\text { 10 } \\
\text { 全 }\end{array}$ & 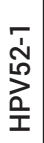 & 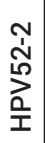 & 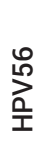 & 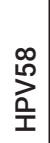 & $\begin{array}{l}\text { 욤 } \\
\text { 몯 }\end{array}$ & $\begin{array}{l}\bullet \\
\stackrel{0}{2} \\
\text { 모 }\end{array}$ & $\begin{array}{l}\text { o } \\
\stackrel{0}{2} \\
\text { 몬 }\end{array}$ & $\begin{array}{l}\frac{1}{0} \\
\frac{0}{0} \\
\frac{\pi}{\pi} \\
\frac{\pi}{0}\end{array}$ \\
\hline
\end{tabular}


Table 4. Sanger sequencing and BLAST search results of a subset of unexpected PCR products.

\begin{tabular}{|c|c|c|}
\hline Reference plasmid & Amplification signal & $\begin{array}{l}\text { Genotype determined by } \\
\text { amplicon sequencing followed } \\
\text { by BLAST search }\end{array}$ \\
\hline \multirow[t]{4}{*}{ HPV31 } & HPV39 & HPV39 \\
\hline & HPV51 & HPV51-1 \\
\hline & HPV52 & HPV52-1 \\
\hline & HPV66 & HPV66 \\
\hline HPV33 & HPV45 & HPV45-1 \\
\hline \multirow[t]{10}{*}{ HPV35 } & HPV16 & OP \\
\hline & HPV18 & HPV18 \\
\hline & HPV31 & HPV31 \\
\hline & HPV51 & HPV51-1 \\
\hline & HPV52 & HPV52-1 \\
\hline & HPV56 & SC \\
\hline & HPV58 & HPV58 \\
\hline & HPV59 & HPV59 \\
\hline & HPV66 & HPV66 \\
\hline & HPV68 & HPV68 \\
\hline \multirow[t]{4}{*}{ HPV39 } & HPV16 & HPV16 \\
\hline & HPV31 & HPV31 \\
\hline & HPV51 & HPV51-1 \\
\hline & HPV66 & HPV66 \\
\hline HPV45-1 & HPV33 & HPV33 \\
\hline HPV45-2 & HPV52 & HPV 52-2 \\
\hline \multirow[t]{2}{*}{ HPV51-1 } & HPV16 & HPV16 \\
\hline & HPV52 & HPV52-1 \\
\hline \multirow[t]{4}{*}{ HPV52-1 } & HPV18 & HPV18 \\
\hline & HPV35 & HPV35 \\
\hline & HPV51 & HPV51-1 \\
\hline & HPV59 & HPV59 \\
\hline HPV52-2 & HPV45 & HPV45-2 \\
\hline \multirow[t]{3}{*}{ HPV56 } & HPV39 & HPV39 \\
\hline & HPV51 & HPV51-1 \\
\hline & HPV52 & HPV52-1 \\
\hline HPV58 & HPV52 & HPV52-1 \\
\hline \multirow[t]{4}{*}{ HPV59 } & HPV35 & HPV35 \\
\hline & HPV51 & HPV51-1 \\
\hline & HPV52 & HPV52-1 \\
\hline & HPV68 & HPV68 \\
\hline
\end{tabular}

and only overnight after the thorough cleaning process was completed was another plasmid purified.

\section{RESULTS \& DISCUSSION}

Unexpected cross-contamination among HR-HPV reference plasmids

For HPV-related research projects, we purchased a set of 17 reference plasmids for 14 HR-HPV genotypes - HPV16, 18, 31, 33, $35,39,45,51,52,56,58,59,66$ and $68-$ among which three genotypes - HPV45, 51 and 52 - each has two sequence variants. To confirm the analytical performance of the CerviHPV test, we performed a standard curve experiment with a tenfold series dilution of the reference plasmids. While the results confirmed excellent linearity and PCR efficiency of CerviHPV test for all HR-HPV genotypes (data not shown), to our surprise an unexpected result occurred at high concentrations of HPV18 plasmid (Table 1 \& Supplementary Figure 1). In addition to the excellent standard curve generated for HPV18 signal, amplification signals were also observed in the 12HPV channel, which also demonstrated good linearity and PCR efficiency that paralleled those of HPV18. This suggested that the source of these unexpected signals was present in the original stock solution of HPV18 plasmid instead of random contamination during setup of the PCR reactions.

Two reasons could account for this result. First, certain 12HPV qPCR probes may crossreact with the HPV18 amplicon sequence within the reference plasmid, as HR-HPV sequences share a high degree of identity. Second, HPV18 plasmid may have been contaminated by unknown $12 \mathrm{HPV}$ plasmid(s). To evaluate these two possibilities, a series of genotyping experiments were performed to determine which 12HPV probe(s) were responsible for the unexpected amplification signal. Remarkably, the three reactions of HR-HPV Genotyping Assay detected amplification signals for nine HR-HPV genotypes - HPV31, $35,39,51,52,56,58,59$ and 66 - at a plasmid concentration of $1.0 \times 10^{8} \mathrm{copies} /$ reaction (Table 2). To determine the identities of the amplified sequences, the PCR products were sequenced with primers specific to the unexpectedly amplified HR-HPV genotypes. As shown in Table 2, the amplification signals for HPV31, 51, 52, 59 and 66 were - 
clearly caused by contamination from the corresponding plasmids. On the other hand, the BLAST search with the sequencing result for the amplified HPV56 signal identified HPV18 itself, consistent with the possibility that the HPV56 probe did crossreact with the HPV18 sequence. However, the possibility of the HPV 56 plasmid contaminating HPV18 plasmid could not be excluded, because the high degree of sequence identity among HR-HPV sequences made it possible that the HPV56 primer used for this sequencing reaction could still sequence HPV18 sufficiently well to overshadow the contaminating HPV56 sequence. For the amplification signals for HPV35, 39 and 58 , the sequencing results were uninterpretable due to overlapping peaks or scrambled chromatograms.

The clear evidence of HPV18 plasmid contamination prompted us to examine whether the other $16 \mathrm{HR}-\mathrm{HPV}$ plasmids were also contaminated by other HR-HPV plasmids using both CerviHPV and genotyping assays. While all plasmids produced amplification signals for the intended HR-HPV types, most of them also amplified signals for other HR-HPV plasmids (Table 3). A representative set of the unexpected $P C R$ products were subjected to Sanger sequencing and BLAST search. While the identities of two out of 44 unexpected PCR products could not be determined due to inadequate sequencing results, BLAST searches identified the remaining 42 to be HR-HPV types of the respective amplification signals but not the template plasmids used, thus confirming cross-contamination among the plasmids (Table 4).

Plasmid cross-contamination occurred before reaching our laboratory

Knowing that the reference plasmids contaminated one another, we wondered when and where such contamination occurred. If it occurred after we received the plasmids, we would need to improve our process to avoid future occurrences. If it occurred prior to their arrival at our laboratory, the cross-contamination must have happened during the production process on the supplier's side. Luckily, we inadvertently created a situation in which we could investigate both possibilities. The 17 plasmids were synthesized de novo, amplified and purified by Supplier A in four

$\begin{aligned} & \text { Table 4. Sanger sequencing and BLAST search results of a subset of } \\
& \text { unexpected PCR products (cont.). }\end{aligned}$
\begin{tabular}{l|l|l|} 
Reference plasmid & Amplification signal & $\begin{array}{l}\text { Genotype determined by } \\
\text { amplicon sequencing followed } \\
\text { by BLAST search }\end{array}$ \\
\hline \multirow{2}{*}{ HPV66 } & HPV16 & HPV16 \\
\cline { 2 - 3 } & HPV31 & HPV31 \\
\cline { 2 - 3 } & HPV39 & HPV39 \\
\cline { 2 - 3 } & HPV51 & HPV51-1 \\
\hline \multirow{2}{*}{ HPV68 } & HPV51 & HPV51-1 \\
\cline { 2 - 3 } & HPV52 & HPV52-1 \\
\cline { 2 - 3 } & HPV56 & HPV56 \\
\cline { 2 - 3 } & HPV58 & HPV58 \\
\hline
\end{tabular}

batches (Table 5). Even though there was extensive cross-contamination among the 12 plasmids from batch No. 2, none of them contaminated the five plasmids of the other three batches. Conversely, the two plasmids from batch No. 1 only contaminated each other but none of the other 15 plasmids, which was also the case for the two plasmids from batch No. 4. The only plasmid from batch No. 3, HPV51-2, was not contaminated by any of the other 16 plasmids; on the other hand, none of the other 16 plasmids were contaminated by HPV51-2 either. Such a pattern strongly suggested that crosscontamination among these plasmids occurred during the production process of Supplier A.

This realization was surprising in that commercial suppliers of biological research reagents usually have more stringent quality control and quality assurance protocols than regular research laboratories. However, even with such stringent protocols, product crosscontamination could still occur. For example, it is still conceivable that a minute amount of one plasmid may be transferred to another plasmid preparation within the same batch through aerosolization due to air flow or vibration [27]. There was also evidence that even HEPA filters could not completely block all DNA aerosols [28].

\section{No cross-contamination among repurified plasmids}

To produce pure HR-HPV plasmids, we transformed competent $E$. coli cells and picked single colonies of the transformants. After overnight culture, new preparations of these plasmids were purified and subsequently subjected to CerviHPV and genotyping tests. The results showed that all the repurified plasmids only produced amplification signals for their respective HR-HPV types (Table 6), further confirming that all the unexpected amplification signals previously observed for the original plasmid preparations (Table 3) were due to cross-contamination among the plasmids rather than cross-reactivity between fluorescent probes and amplicon sequences of different HR-HPV types.

\section{Another batch of cross-contaminated} plasmid preparations from Supplier A Such a revelation prompted us to examine another set of reference plasmids for 13 bacterial diarrhea-causing virulence genes. They were synthesized by Supplier A in two batches - stx1, stx2, eae, bfpB, stp and invE in the first batch and escV, sth, It, aggR, ast $A$, pic and $i p a H$ in the second batch. With all 13 plasmids as templates, we performed seven qPCR reactions, each targeting one of the seven plasmids from batch no. 2 . The results showed that every one of the seven plasmids from batch no. 2 produced amplification signals for all seven genes encoded by the plasmids from the same batch (Supplementary Table 1). In contrast, none of the six plasmids from batch no. 1 produced any amplification signal for the seven genes encoded by the plasmids from batch no. 2 . Unlike the highly homologous HR-HPV sequences, the low sequence identity among the 13 diarrhea-causing virulence genes made it unlikely that the unintended amplification signals were the results of cross-reactivity between the primers and 


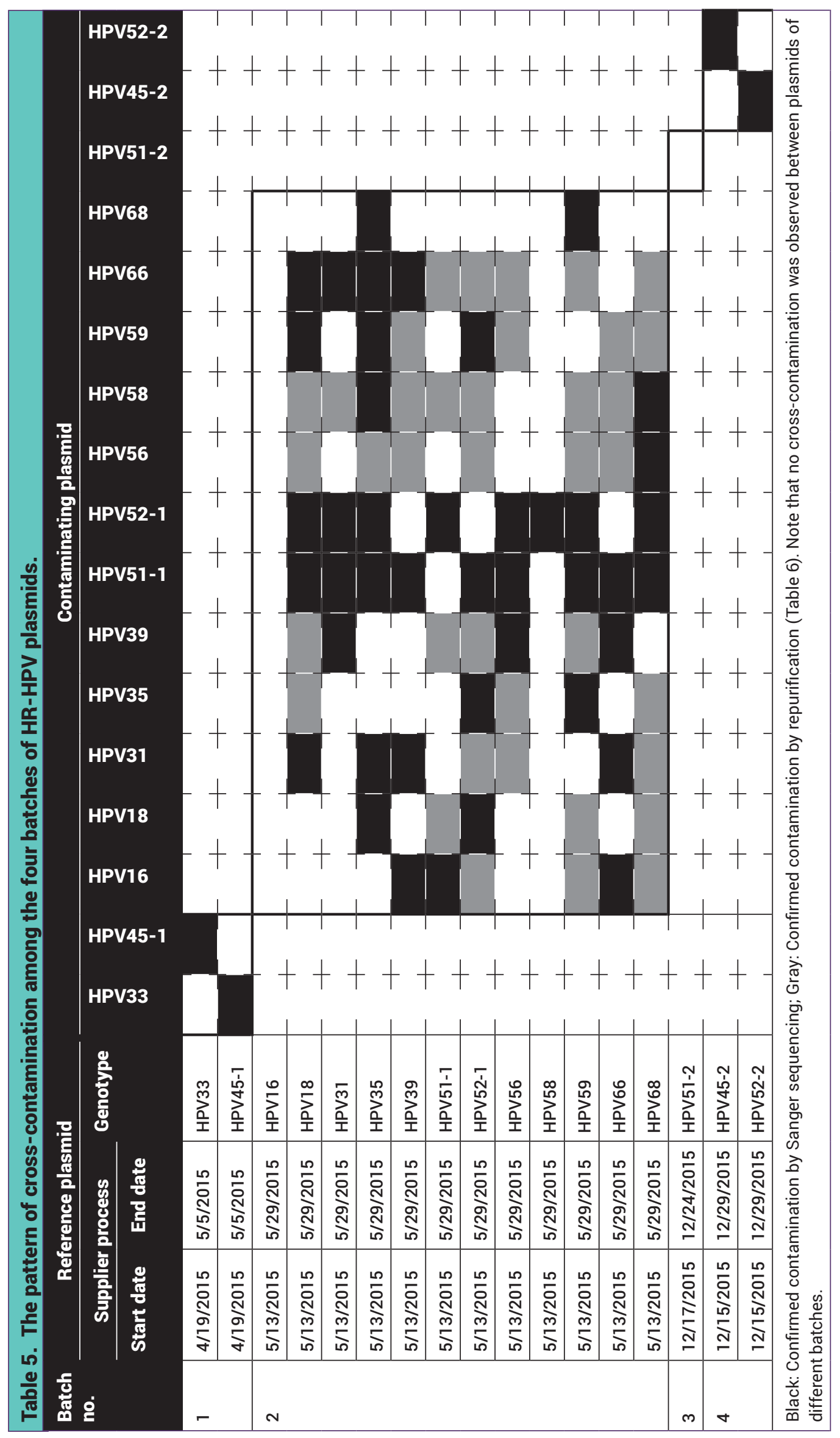




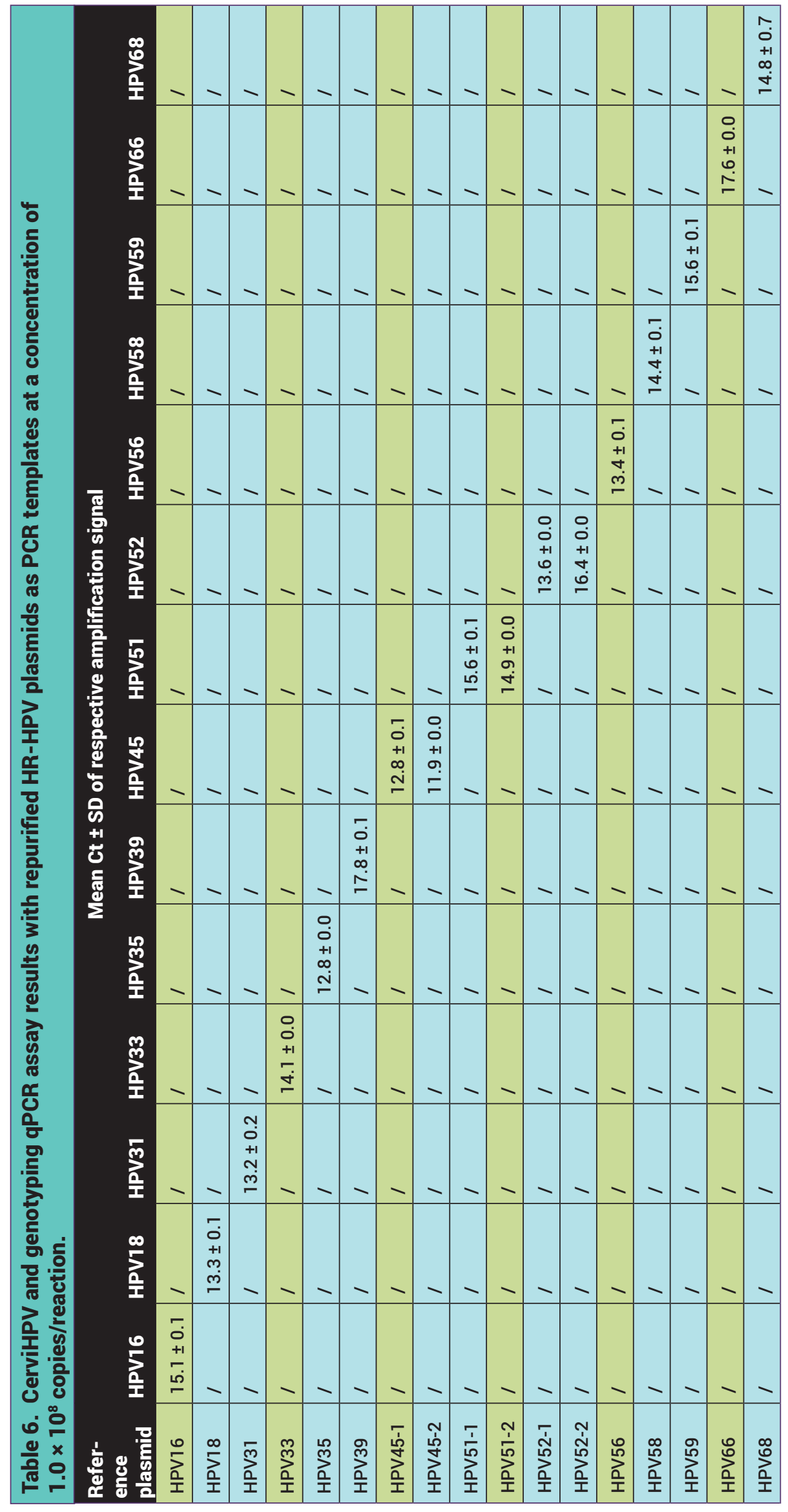

probe of one gene and the plasmid sequence of another. This was confirmed for nine representative cases by Sanger sequencing of the PCR products and subsequent BLAST searches (Supplementary Table 1). Taken together, the above results made it extremely unlikely that the cross-contamination among the plasmids from batch no. 2 occurred in our laboratory, thus pointing to the conclusion that they also occurred during the production process of Supplier A. Indeed, qPCR reactions with repurified plasmids did not produce any unintended amplification signal (data not shown).

Cross-contamination between plasmids purchased from Supplier B

Cross-contamination among plasmids purchased at the same time from the same supplier was not specific to Supplier A. We purchased two plasmids in a single order from Supplier B. They contained cDNA sequences for human IL7B and CXCL8 genes. Whereas no qPCR amplification signal for CXCL8 was observed with IL7B plasmid, CXCL8 plasmid did produce an amplification signal for ILIB, which was confirmed to be the result of cross-contamination, as it disappeared from repurified CXCL8 plasmid (Supplementary Table 2).

In the present study, we provided clear evidence that plasmids purchased from commercial suppliers of biological research reagents may be cross-contaminated by other plasmids within the same batch during the production process, which has happened to two different commercial suppliers. To our knowledge, our work was the first study to demonstrate such crosscontamination unequivocally, which might have occurred even more often in regular research laboratories. Therefore, if our experience was any guide, the purity of any biological reagents obtained from outside sources should not be taken for granted. If absolute purity is required, it will be prudent to implement quality control processes to determine if cross-contamination has occurred. For plasmids, genomic DNA and bacteria strains, a simple set of specific PCR reactions similar to what we used in this study would be able to identify such contamination. And if cross-contamination does occur, there may be ways to eliminate the contamination and produce pure 
reagents. In the case of plasmids, the repurification protocol and the subsequent confirmation test described in this work should be sufficient. The key to this protocol is the thorough cleaning of all equipment used for plasmid purification before and after purification, as well as purifying only one plasmid each day. Similarly, for bacterial strains, simple restreaking on agar plates and picking single colonies should be able to isolate the desired strain from contaminating bacteria.

Based on our experience, researchers obtaining plasmids from outside sources, either industrial or academic, should be mindful of the possibility of contamination by other plasmids. If absolute plasmid purity is required for the project at hand, it is imperative to implement a standard protocol to repurify every outside plasmid due to the possibility of contamination by unknown plasmids, for which the researcher may have no way of testing. For example, plasmids synthesized de novo by commercial suppliers may be contaminated by same-batch plasmids synthesized for other customers. On the other hand, commercial suppliers should improve quality assurance and quality control protocols for their production processes to minimize cross-contamination among their products.

\section{SUPPLEMENTARY DATA}

To view the supplementary data that accompany this paper please visit the journal website at: www.future-science. com/doi/suppl/10.2144/btn-2019-0018

\section{AUTHOR CONTRIBUTIONS}

JS, YT and YM initially discovered the issue of cross-contamination among plasmids. GZ, YM, MZ conceived the study. JS, YD, ZW and $G Z$ performed the experiments. JS, YT, GZ and YM analyzed the data. JS, YT, GZ, YM and MZ drafted the manuscript. All authors approved the final manuscript.

\section{ACKNOWLEDGMENTS}

The authors would like to acknowledge the excellent technical assistance by Ms Yanjun Yang.

\section{FINANCIAL \& COMPETING}

\section{INTERESTS DISCLOSURE}

This work was supported by the 'Hundred Talents Program' of Chinese Academy of Sciences (grant no. Y421021102, grant no. Y521051102), Key Technologies R\&D Program for Social Development of Jiangsu Province (grant no. BE2016685), Suzhou Leading Talent Project (grant no. ZXL2014128) and New Medical Devices and Pharmaceuticals Fund of Suzhou (grant no. ZXY201430). The authors have no other relevant affiliations or financial involvement with any organization or entity with a financial interest in or financial conflict with the subject matter or materials discussed in the manuscript apart from those disclosed.

No writing assistance was utilized in the production of this manuscript.

\section{OPEN ACCESS}

This work is licensed under the AttributionNonCommercial-NoDerivatives 4.0 Unported License. To view a copy of this license, visit http://creativecommons.org/licenses/ by-nc-nd/4.0/

\section{REFERENCES}

1. Kado $\mathrm{Cl}$. Historical events that spawned the field of plasmid biology. Microbiol. Spectr. 2(5), (2014).

2. Nathans D, Smith HO. Restriction endonucleases in the analysis and restructuring of DNA molecules. Ann. Rev. Biochem. 44(1), 273-293 (1975).

3. Loenen WAM, Dryden DTF, Raleigh EA, Wilson GG, Murray NE. Highlights of the DNA cutters: a short history of the restriction enzymes. Nucleic Acids Res. 42(1), 3-19 (2014).

4. Lobban PE, Kaiser AD. Enzymatic end-to-end joining of DNA molecules. J. Mol. Biol. 78(3), 453-460 (1973).

5. Kaufman RJ. Overview of vector design for mammalian gene expression. Mol Biotechnol. 16(2), 151-160 (2000).

6. Stepanenko AA, Heng HH. Transient and stable vector transfection: Pitfalls, off-target effects, artifacts. Mutat. Res. Rev. Mutat. 773, 91-103 (2017).

7. Athanasopoulos T, Munye MM, Yanez-Munoz RJ. Nonintegrating gene therapy vectors. Hematol. Oncol. Clin. N. 31(5), 753-770 (2017).

8. Prazeres DMF, Monteiro GA. Plasmid biopharmaceuticals. Microbiol. Spectr. 2(6), (2014).

9. Hobernik D, Bros M. DNA vaccines - how far from clinical use? Int. J. Mol. Sci. 19(11), 3605 (2018)

10. Ahmad N, Mukhtar Z. Genetic manipulations in crops: challenges and opportunities. Genomics 109(5-6), 494-505 (2017)

11. Cui WJ, Han LC, Suo FY, Liu ZM, Zhou L, Zhou ZM. Exploitation of Bacillus subtilis as a robust workhorse for production of heterologous proteins and beyond. World J. Microb. Biot. 34(10), (2018).

12. Khorana HG. Nucleic acid synthesis. Pure Appl. Chem. 17, 349-381 (1968)

13. Stemmer WP, Crameri A, Ha KD, Brennan TM, Heyneker $\mathrm{HL}$. Single-step assembly of a gene and entire plasmid from large numbers of oligodeoxyribonucleotides. Gene 164(1), 49-53 (1995).

14. Kosuri S, Church GM. Large-scale de novo DNA synthesis: technologies and applications. Nat. Methods 11(5), 499-507 (2014)

15. Sarepta Therapeutics Inc. Sarepta Therapeutics announces that phase 1/2a Duchenne Muscular Dystrophy (DMD) micro-dystrophin gene therapy trial placed on clinical hold due to an out-of-specification production lot; no observed safety events (2018). http://investorrelations.sarepta.com/news-releases/ news-release-details/sarepta-therapeutics-announces-phase-12a-duchenne-muscular

16. van der Heijden I, Gomez-Eerland R, van den Berg JH et al. Transposon leads to contamination of clinical pDNA vaccine. Vaccine 31(32), 3274-3280 (2013).

17. Khodakov D, Wang CY, Zhang DY. Diagnostics based on nucleic acid sequence variant profiling: PCR, hybridization, and NGS approaches. Adv. Drug Deliver. Rev. 105, 3-19 (2016).

18. Lo YMD, Mehal WZ, Fleming KA. False-positive results and the polymerase chain-reaction. Lancet 2(8612), 679-679 (1988).

19. Czurda S, Smelik S, Preuner-Stix S, Nogueira F, Lion T. Occurrence of fungal DNA contamination in PCR reagents: approaches to control and decontamination J. Clin. Microbiol. 54(1), 148-152 (2016).

20. Ruella $M, X u$ J, Barrett DM et al. Induction of resistance to chimeric antigen receptor T cell therapy by transduction of a single leukemic B cell. Nat. Med. 24(10), 1499-1503 (2018).

21. Bagutti C, Alt M, Schmidlin M, Vogel G, Vogeli U, Brodmann P. Detection of adeno- and lentiviral (HIV1) contaminations on laboratory surfaces as a tool for the surveillance of biosafety standards. J. Appl. Microbiol. 111(1), 70-82 (2011)

22. Wu YP, Wu JY, Zhang ZH, Cheng C. DNA decontamination methods for internal quality management in clinical PCR laboratories. J. Clin. Lab. Anal. 32(3), (2018).

23. Pruvost M, Grange T, Geigl EM. Minimizing DNA contamination by using UNG-coupled quantitative real-time PCR on degraded DNA samples: application to ancient DNA studies. Biotechniques 38(4), 569-575 (2005).

24. Goldsmith M, Kiss C, Bradbury ARM, Tawfik DS. Avoid ing and controlling double transformation artifacts. Protein Eng. Des. Sel. 20(7), 315-318 (2007).

25. Zhao G, Tian Y, Du Y et al. Comparison of CerviHPV and Hybrid Capture $2 \mathrm{HPV}$ tests for detection of high-risk HPV infection in cervical swab specimens. Diagn. Cytopathol. 47(5), 439-444 (2019).

26. National Center for Biotechnology Information. Basic local alignment search tool. https://blast.ncbi.nlm.nih. gov/Blast.cgi

27. Scherczinger CA, Ladd C, Bourke MT et al. A systematic analysis of PCR contamination. J. Forensic Sci. 44(5), 1042-1045 (1999)

28. Morono $\mathrm{Y}$, Hoshino $\mathrm{T}$, Terada $\mathrm{T}$ et al. Assessment of capacity to capture DNA aerosols by clean Filters for molecular biology experiments. Microbes Environ. 33(2), 222-226 (2018) 